\title{
Indivisibility of class numbers of imaginary quadratic function fields
}

by

\author{
Dongho Byeon (Seoul)
}

1. Introduction. Let $p$ be an odd prime number, $q$ a power of $p$, and $\mathbb{F}_{q}$ the finite field of cardinality $q$. Let $T$ be an indeterminate and $K=\mathbb{F}_{q}(T)$ the rational function field. Let $A=\mathbb{F}_{q}[T]$ and $A^{(1)}$ be the set of all non-zero monic polynomials in $A$.

There have been many works on the divisibility of class numbers of function fields $F$ over $K$. For example, Friesen [3] and Cardon and Murty [1] proved that there are infinitely many real and imaginary, respectively, quadratic extensions $F$ over $K$ such that the class number of $F$ is divisible by $l$, which is a function field analogue of the well-known result on the quadratic number fields.

However, much less is known on indivisibility. In [6], Kimura proved that there are infinitely many quadratic extensions $F$ over $K$ such that the class number of $F$ is not divisible by 3 . For an odd prime number $l$, Ichimura [5] constructed infinitely many imaginary quadratic extensions $F$ over $K$ such that the class number of $F$ is not divisible by $l$, when the order of $q \bmod l$ in the multiplicative group $(\mathbb{Z} / l \mathbb{Z})^{*}$ is odd or $l=p$.

In this paper, we shall prove the following theorem.

TheOREM 1.1. Let $l$ be an odd prime number. Then there are infinitely many imaginary quadratic extensions $F$ over $K$ such that the class number of $F$ is not divisible by $l$.

Theorem 1.1 is a function field analogue of Hartung's work [4] on imaginary quadratic number fields. To prove it, following Hartung's idea in [4], we shall use the class number relation over function fields, due to $\mathrm{Yu}[8]$.

2000 Mathematics Subject Classification: Primary 11R58; Secondary 11R11, 11R29.

Key words and phrases: class number, function field.

This work was supported by KRF-2005-070-C00004.

The author also holds joint appointment in the Research Institute of Mathematics, Seoul National University. 
REMARK. In the number field case, the Cohen-Lenstra heuristics implies that if $l$ is an odd prime number, then the probability that $l$ does not divide the class number of an imaginary quadratic number field is

$$
\prod_{i=1}^{\infty}\left(1-1 / l^{i}\right) \text {. }
$$

In the function field case, Lee [7, Section 3.3] shows that Friedman and Washington's conjectures [2] for the function field analogue of the CohenLenstra heuristics imply that if $l(\neq p)$ is an odd prime number, then the probability that $l$ does not divide the class number of an imaginary quadratic function field is also $\prod_{i=1}^{\infty}\left(1-1 / l^{i}\right)$.

2. Class number relation. For details, we refer to the paper of $\mathrm{Yu}$ [8]. Let $D \in A$ be a fundamental discriminant. Let $F=K(\sqrt{D})$ be the quadratic extension over $K=\mathbb{F}_{q}(T)$ and $\mathcal{O}_{D f^{2}}=A+A \sqrt{D f^{2}}$ the order of conductor $f \in A^{(1)}$ in $F$. The order of the finite group $\operatorname{Pic}\left(\mathcal{O}_{D f^{2}}\right)$ is called the class number of discriminant $D f^{2}$ and is denoted by $h\left(D f^{2}\right)$.

From now on, we assume that $F=K(\sqrt{D})$ is imaginary, i.e., the place $\infty$ of $K$ does not split in $F$. We also say that $D$ and $D f^{2}$ are imaginary discriminants. Then we can define $\omega\left(D f^{2}\right):=\sharp \mathcal{O}_{D f^{2}}^{*} /(q-1)$ and $h^{\prime}\left(D f^{2}\right):=$ $h\left(D f^{2}\right) / \omega\left(D f^{2}\right)$. Let $\chi_{D}$ be the usual Kronecker character satisfying for prime $P \in A^{(1)}, \chi_{D}(P)=1$ if $P$ splits in $F, \chi_{D}(P)=0$ if $P$ ramifies in $F$, and $\chi_{D}(P)=-1$ otherwise. For an element $x \in A$, we let $|x|:=q^{\operatorname{deg} x}$.

Then for any fundamental imaginary discriminant $D$ and conductor $f$, we have

$$
h^{\prime}\left(D f^{2}\right)=h^{\prime}(D)|f| \prod_{P \mid f}\left(1-\frac{\chi_{D}(P)}{|P|}\right)
$$

where the product runs over primes $P \in A^{(1)}$ dividing $f$. We define the Hurwitz class number $H\left(D f^{2}\right)$ as

$$
H\left(D f^{2}\right):=\sum_{\substack{f^{\prime} \in A^{(1)} \\ f^{\prime} \mid f}} h^{\prime}\left(D f^{\prime 2}\right) .
$$

$\mathrm{Yu}$ obtained the following class number relation.

Theorem 2.1 (Yu $[8])$. For any $m$ in $A^{(1)}$,

$$
\sum_{\substack{t \in A \\ \mu \in K^{*} / K^{* 2}}} H\left(t^{2}-\mu m\right)=\sum_{\substack{d \in A^{(1)} \\ d \mid m}} \max (|d|,|m / d|)-\sum_{\substack{d \in A^{(1)} \\ d \mid m \\ \operatorname{deg} d=1 / 2 \operatorname{deg} m}}|m|^{-1 / 2} \frac{|m|-\left|m-d^{2}\right|}{q-1},
$$


where the first sum runs over all pairs $(t, \mu) \in A \times K^{*} / K^{* 2}$ such that $t^{2}-\mu m$ is an imaginary discriminant or $t^{2}-\mu m=0$.

3. Proof of Theorem 1.1. For $l=p$, Ichimura already constructed infinitely many imaginary quadratic extensions $F$ over $K$ such that the class number of $F$ is not divisible by $l$ (see Theorem 3 in [5]). So in this section we consider the case $l \neq p$. We can choose $m$ satisfying:

(i) $m$ is a prime in $A^{(1)}$ with odd degree $M$,

(ii) $\chi_{D}(m)=-1$ for all imaginary fundamental discriminants $D$ of degree $\leq N$.

Then from the class number relation in Theorem 2.1 and (i), we have

$$
\sum_{\substack{t \in A \\ \mu \in K^{*} / K^{* 2}}} H\left(t^{2}-\mu m\right)=2 q^{M} .
$$

Since $l \neq p$, there is a pair $(t, \mu) \in A \times K^{*} / K^{* 2}$ such that

$$
H\left(t^{2}-\mu m\right) \not \equiv 0(\bmod l) \text {. }
$$

We can write

$$
t^{2}-\mu m=D_{t, \mu} f^{2}
$$

for some imaginary fundamental discriminant $D_{t, \mu}$ and conductor $f$. By the definition of $h^{\prime}$ and the Hurwitz class number, we have

$$
h\left(D_{t, \mu}\right) \not \equiv 0(\bmod l) .
$$

From the condition (ii), the degree of $D_{t, \mu}>N$. Since $N$ can be arbitrarily large, there are infinitely many imaginary fundamental discriminants $D$ whose class number $h(D)$ is not divisible by $l$.

Acknowledgements. The author thanks the referee for some helpful suggestions.

\section{References}

[1] D. A. Cardon and M. R. Murty, Exponents of class groups of quadratic function fields over finite fields, Canad. Math. Bull. 44 (2001), 398-407.

[2] E. Friedman and L. Washington, On the distribution of divisor class groups of curves over a finite field, in: Théorie des nombres (Québec, PQ, 1987), de Gruyter, Berlin, 1989, 227-239.

[3] C. Friesen, Class number divisibility in real quadratic function fields, Canad. Math. Bull. 35 (1992), 361-370.

[4] P. Hartung, Proof of the existence of infinitely many imaginary quadratic fields whose class numbers are not divisible by three, J. Number Theory 6 (1976), 276-278.

[5] H. Ichimura, Quadratic function fields whose class numbers are not divisible by three, Acta Arith. 91 (1999), 181-190. 
[6] I. Kimura, On class numbers of quadratic extensions over function fields, Manuscripta Math. 97 (1998), 81-91.

[7] Y. Lee, Cohen-Lenstra heuristics and the Spiegelungssatz, Dissertation, Brown Univ., 1999.

[8] J. K. Yu, A class number relation over function fields, J. Number Theory 54 (1995), 318-340.

Department of Mathematics

Seoul National University

Seoul 151-747, Korea

E-mail: dhbyeon@math.snu.ac.kr

Received on 4.8.2007

and in revised form on 29.2.2008 